\title{
PROFESIONALES DE LA SALUD PARA EL NUEVO SIGLO: TRANSFORMANDO LA EDUCACIÓN PARA FORTALECER LOS SISTEMAS DE SALUD EN UN MUNDO INTERDEPENDIENTE
}

\author{
HEALTH PROFESSIONALS FOR A NEW CENTURY: \\ TRANSFORMING EDUCATION TO STRENGTHEN HEALTH SYSTEMS \\ IN AN INTERDEPENDENT WORLD
}

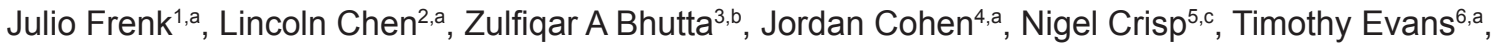
Harvey Fineberg ${ }^{7, a}$, Patricia J. García ${ }^{8, a}$, Yang Ke ${ }^{9, a}$, Patrick Kelley ${ }^{9, a}$, Barry Kistnasamy ${ }^{10, a}$, Afaf Meleis ${ }^{11, b}$, David Naylor ${ }^{12, a}$, Ariel Pablos-Mendez ${ }^{13, a}$, Srinath Reddy ${ }^{14, a}$, Susan Scrimshaw ${ }^{15, b}$, Jaime Sepulveda ${ }^{16, a}$,

David Serwadda ${ }^{17, a}$, Huda Zurayk ${ }^{18, b}$
\end{abstract}

\section{Descripción del Problema}

Hace 100 años, una serie de estudios sobre la educación de los profesionales de la salud, liderado por el informe Flexner de 1910, introdujeron reformas innovadoras. Mediante la integración de la ciencia moderna en el currículo de las facultades de las universidades, estas reformas equiparon a los profesionales de la salud con el conocimiento que contribuyó a duplicar la esperanza de vida durante el siglo XX.

A inicios del siglo XXI, sin embargo, no todo está bien, persisten evidentes vacíos y desigualdades en salud tanto entre países como dentro de ellos, haciendo evidente la falla colectiva de compartir de manera equitativa los dramáticos avances en salud. Al mismo tiempo, se avecinan grandes retos, es decir, nuevos riesgos infecciosos, medioambientales y conductuales, que, en un momento de rápidas transiciones demográficas y epidemiológicas, amenazan la seguridad de la salud de todos. Los sistemas de salud del mundo están luchando para mantenerse en el nivel requerido, mientras que se hacen más complejos y costosos, poniendo exigencias adicionales sobre los trabajadores de la salud.

La educación profesional no se ha desarrollado al ritmo de estos retos, fundamentalmente por contar con un currículo fragmentado, obsoleto y estático que produce graduandos mal equipados. Los problemas son sistémicos: una descoordinación entre las competencias y las necesidades de los pacientes y de la población; deficiente trabajo en equipo; una persistente estratificación de los géneros en el estatus profesional; un enfoque técnico limitado sin un entendimiento conceptual más amplio; encuentros episódicos en lugar de un cuidado de salud continuo; una predominante orientación hacia los hospitales a expensas de la atención primaria; desbalances cuantitativos y cualitativos en el mercado laboral profesional y un débil liderazgo para mejorar el rendimiento de los sistemas de salud. Loables esfuer-

\footnotetext{
Harvard School of Public Health. Boston, Massachusetts, Estados Unidos de Norteamérica.

China Medical Board. Cambridge, Massachusetts, Estados Unidos de Norteamérica.

Aga Khan University. Karachi, Pakistan.

George Washington University Medical Center. Washington, DC, Estados Unidos de Norteamérica.

Independent member of House of Lords. London, Reino Unido.

James P Grant School of Public Health. Dhaka, Bangladesh.

US Institute of Medicine. Washington, DC,Estados Unidos de Norteamérica.

Facultad de Salud Pública y Administración, Universidad Peruana Cayetano Heredia. Lima, Perú.

Peking University Health Science Centre. Beijing, China.

National Health Laboratory Service. Johannesburg, Sudáfrica.

School of Nursing, University of Pennsylvania. Philadelphia, Estados Unidos de Norteamérica.

University of Toronto. Toronto, Canada.

3 The Rockefeller Foundation. New York, Estados Unidos de Norteamérica.

14 Public Health Foundation of India. New Delhi, India.

15 The Sage Colleges. Troy, MI, Estados Unidos de Norteamérica.

16 Bill \& Melinda Gates Foundation. Seattle, Estados Unidos de Norteamérica.

17 Makarere University School of Public Health. Kampala, Uganda.

18 Centre for Research on Population and Health, Faculty of Health Sciences, American University of Beirut. Beirut, Líbano.

a Médico; ' $\mathrm{PhD} ;{ }^{\mathrm{c}} \mathrm{KCB}$.
} 
zos para enfrentar estas deficiencias han fracasado en su mayoría, en parte debido al denominado "tribalismo" de las profesiones, es decir, la tendencia de las diversas profesiones para actuar en aislamiento o incluso en franca competencia con las otras.

Es necesario y oportuno un rediseño de la educación profesional en salud, en vista de las oportunidades de aprendizaje mutuo y soluciones conjuntas que ofrece la interdependencia global causada por la aceleración de los flujos de conocimiento, tecnologías y financiamiento a través de las fronteras y la migración tanto de profesionales como de pacientes. Lo que claramente se necesita es reexaminar seriamente, y a fondo, la educación profesional en salud, tal como se hizo hace un siglo.

Es por ello que esta Comisión, que consta de 20 líderes profesionales y académicos de diversos países, se reunió para desarrollar una visión compartida y una estrategia en común para la educación en medicina, enfermería y salud pública, que llegue más allá de los confines de las fronteras nacionales y de los silos de las profesiones individuales. La Comisión adoptó una visión global, una perspectiva multiprofesional y un enfoque de sistemas. Este marco integral considera las conexiones entre la educación y los sistemas de salud. Se centra en las personas como coproductores y como generadores de las necesidades y demandas en ambos sistemas. Mediante la interacción con el mercado laboral, la provisión de servicios educativos genera la producción de una fuerza laboral educada que satisface la demanda de profesionales que trabajen en el sistema de salud. Para lograr un efecto positivo en los indicadores de salud, el subsistema de educación profesional deberá diseñar nuevas estrategias instructivas e institucionales.

\section{Principales hallazgos}

A nivel mundial, 2420 escuelas de medicina, 467 escuelas o departamentos de salud pública y un número indeterminado de instituciones postsecundarias de educación en enfermería preparan alrededor de 1 millón de nuevos médicos, enfermeras, obstetrices y profesionales de la salud pública cada año. Las graves deficiencias institucionales se ven exacerbadas por una mala distribución de los recursos, tanto entre los países como dentro de ellos mismos.

Cuatro países (China, India, Brasil y EE.UU.) tienen cada uno más de 150 escuelas de medicina, mientras que 36 países no tienen ni una escuela de medicina; en tanto, en el África subsahariana, 26 países tienen una o ninguna escuela de medicina. En vista de estos desbalances, no es sorpresa que el número de escuelas de medicina no se relacione ni con el tamaño de la población del país ni con la carga nacional de enfermedades.

El gasto global total para la educación profesional en salud bordea los U.S. $\$ 100$ billones por año, nuevamente con grandes disparidades entre países. Este monto es menor al $2 \%$ de los gastos en salud a nivel mundial, lo cual resulta lamentablemente modesto para una industria de intenso trabajo y dirigida por el talento. El costo promedio por graduando es de $\$ 113000$ para los estudiantes de medicina y de \$46 000 para las enfermeras, con los más altos costos unitarios en Norteamérica y los más bajos en China.

Los sistemas administrativos, de acreditación y de enseñanza son débiles y se practican desigualmente alrededor del mundo. Nuestro análisis ha demostrado la escasez de información e investigación respecto a la educación profesional en salud. Pese a que muchas instituciones educativas en todas las regiones han lanzado iniciativas innovadoras, se dispone de poca evidencia robusta con respecto a la efectividad de dichas reformas.

\section{Reformas para un segundo siglo}

Tres generaciones de reformas educativas caracterizan el progreso durante el siglo pasado. La primera generación, lanzada a inicios del siglo XX, planteó un currículo basado en la ciencia. Llegando a la mitad del siglo, la segunda generación introdujo innovaciones instructivas mediante un currículo basado en la resolución de problemas. Ahora, se necesita una tercera generación con un currículo basado en sistemas para mejorar el rendimiento de los sistemas de salud mediante la adaptación de las competencias profesionales esenciales a contextos específicos, pero sobre la base del conocimiento global.

Para avanzar con las reformas de la tercera generación, la Comisión presenta una visión: todos los profesionales de la salud en todos los países deberán ser entrenados para movilizar el conocimiento y comprometerse al razonamiento crítico y a una conducta ética de modo que se hagan competentes para participar en los sistemas de salud centrados en el paciente y la población como miembros activos de los equipos de salud que proveen una respuesta local y están conectados globalmente. El propósito fundamental es asegurar la cobertura universal de los servicios integrales de alta calidad que son esenciales para mejorar las oportunidades de igualdad en salud entre los países y dentro de ellos.

Materializar esta visión requerirá una serie de reformas instructivas e institucionales, las cuales deberán ser guiadas por los dos resultados que se proponen: un aprendizaje transformacional y una interdependencia 
en la educación. Entendemos un aprendizaje transformacional como la cúspide de tres niveles sucesivos, que van desde un aprendizaje informativo hacia un aprendizaje formativo y hacia un aprendizaje transformacional. Aprendizaje informativo se refiere a adquirir conocimiento y habilidades; su propósito es producir expertos. Aprendizaje formativo se refiere a socializar a los estudiantes basándose en valores; su propósito es producir profesionales. El aprendizaje transformacional se refiere a desarrollar cualidades de liderazgo; su propósito es producir reales agentes de cambio. Una educación efectiva construye cada nivel sobre el nivel previo. Como parte de un valioso resultado, un aprendizaje transformacional involucra tres cambios fundamentales: un alejamiento de la memorización de hechos en dirección a la búsqueda, el análisis y la síntesis de la información que conduzca a la toma de decisiones; el reemplazo de la búsqueda de credenciales profesionales por el logro de competencias esenciales para un trabajo en equipo efectivo dentro de los sistemas de salud; y de la adopción sin crítica de modelos educativos hacia una adaptación creativa de recursos globales para enfrentar prioridades locales. La interdependencia es un elemento clave dentro de un enfoque de sistemas dado que pone en relieve las formas en que los diversos componentes interactúan. Como resultado deseable, la interdependencia en educación también involucra tres cambios fundamentales: el movimiento de una educación aislada hacia una educación y sistemas de salud armonizados; el cambio de instituciones independientes hacia redes, alianzas y consorcios y el paso de preocupaciones institucionales que buscan una mejora interna hacia el aprovechamiento de los flujos globales de contenidos educativos, recursos de enseñanza e innovaciones.

Un aprendizaje transformacional es el resultado que se propone a partir de las reformas instructivas; la interdependencia en educación deberá resultar de reformas institucionales. Sobre la base de estas nociones esenciales, la Comisión ofrece una serie de recomendaciones específicas para mejorar el rendimiento de los sistemas. Las reformas instructivas deberán: adoptar estrategias dirigidas a lograr competencias dentro del diseño instructivo; adaptar estas competencias a las rápidamente cambiantes condiciones locales recurriendo a los recursos globales; promover una educación interprofesional y transprofesional que vaya más allá de los silos profesionales realzando a la vez las relaciones de colaboración no-jerárquicas dentro de equipos efectivos; explotar el poder de la tecnología de la información para el aprendizaje; reforzar los recursos educativos, con especial énfasis en el desarrollo de los docentes de las facultades; y promover un nuevo profesionalismo que utilice competencias como criterio objetivos para la clasificación de los profesionales de la salud y que desarrolle un conjunto de valores en común centrados en la responsabilidad social. Las reformas institucionales deberán establecer dentro de cada país mecanismos conjuntos de educación y planeamiento en salud que tomen en cuenta dimensiones cruciales, tales como el origen social, la distribución de edades y la composición por géneros, de la fuerza de trabajo en salud; expandir los centros académicos para hacerlos sistemas académicos que incluyan redes de hospitales y unidades de atención primaria; enlazarse dentro en redes globales, alianzas y consorcios; y alimentar una cultura de cuestionamiento crítico.

La puesta en práctica de estas reformas encontrará muchas barreras. Nuestras recomendaciones, por lo tanto, requieren una serie de acciones que faciliten el proceso. En primer lugar, el más amplio compromiso de los líderes en todos los niveles -local, nacional y globalserá crucial para alcanzar las reformas y resultados propuestos. El liderazgo tiene que provenir del interior de las comunidades académicas y profesionales, pero deberá estar respaldada por los líderes políticos dentro del gobierno y la sociedad. Segundo, las actuales deficiencias financieras deberán ser superadas mediante una sustancial expansión de las inversiones en la educación profesional en salud de todas las fuentes: públicas, privadas, de soporte al desarrollo y fundaciones. Tercero, los mecanismos de responsabilidad administrativa, que incluyan una acreditación en responsabilidad social, deberán ser reforzados para asegurar los mejores resultados posibles para cualquier nivel de financiamiento. Por último, se deberá fortalecer el aprendizaje compartido mediante el uso de mediciones (indicadores), evaluaciones e investigación para construir el conocimiento base respecto a qué innovaciones funcionan y en qué circunstancias.

Los profesionales de la salud han realizado enormes contribuciones a la salud y el desarrollo durante el siglo pasado, pero la complacencia solo perpetuará la inefectiva aplicación de las estrategias educativas del siglo $X X$ que no son apropiadas para enfrentar los retos del siglo XXI. Por lo tanto, hacemos un llamado para un movimiento social global de todos aquellos participantes -educadores, estudiantes y jóvenes trabajadores de la salud, organizaciones profesionales, universidades, organismos no gubernamentales, agencias internacionales, donantes y fundaciones- que pueden propulsar la acción sobre esta visión y estas recomendaciones para promover un nuevo siglo de educación profesional transformativa. El resultado será tener sistemas de salud más equitativos y de mejor rendimiento que los actuales, que traigan los consecuentes beneficios para los pacientes y las poblaciones de cualquier lugar dentro de nuestro mundo interdependiente. 


\section{AGRADECIMIENTOS}

Los miembros de la comisión se sienten complacidos de reconocer la contribución del equipo de investigación, compuesto por Catherine M. Michaud, Ananda S Bandyopadhyay, Chenhui Liu, Ruvandhi Nathavitharana, Stanislava Nikolova, Sonali Vaid, y Leana S Wen. Ellos también desean agradecer a dos revisores independientes, Anita Berlin y Kenji Shibuya, por sus valiosos comentarios sobre al manuscrito remitido.

\section{Contribuciones de Autoría}

Todos los comisionados contribuyeron con los conceptos, recomendaciones, redacción y edición del informe,

\section{Panel 9: reformas propuestas}

Las reformas instructivas deberán incluir el rango completo desde la admisión hasta la graduación, para generar un cuerpo diverso de estudiantes con un currículo basado en competencias que, a través del uso creativo de las tecnologías de la información (TIC), prepara estudiantes para la realidades de trabajo en equipo, para desarrollar rutas flexibles de carrera que están basadas en el espíritu y los deberes de un nuevo profesionalismo.

1. Adopción de un currículo basado en competencias que responda a las rápidamente cambiantes necesidades en lugar de estar dominado por un trabajo estático de curso. Las competencias deberán ser adaptadas a contextos locales y determinados por los participantes nacionales, mientras se domina el conocimiento y experiencias globales. Simultáneamente, los presentes vacíos deberán ser llenados en el rango de competencias que se requiere para lidiar con los retos del siglo XXI comunes a todos los países-ej., la respuesta a las amenazas a la seguridad de salud global o el manejo de cada vez más complejos sistemas de salud.

2. Promoción de educación interprofesional y transprofesional que derribe los silos profesionales y a la vez realce las relaciones colaborativas y no jerárquicas en equipos efectivos. Junto con las habilidades técnicas específicas, la educación interprofesional se deberá enfocar en competencias genéricas contrapuestas, tales como las habilidades analíticas (para el uso efectivo tanto de la evidencia como de la deliberación ética en la toma de decisiones), liderazgo y capacidades de administración (para un eficiente manipuleo de los escasos recursos en condiciones de incertidumbre) y habilidades de comunicación (para la movilización de todos los participantes, incluyendo pacientes y poblaciones).

3. Explotación del poder de las TIC para aprender mediante el desarrollo de evidencia, capacidad para la recolección y análisis de data, simulación y exámenes, aprendizaje a distancia, conectividad colaborativa y manejo del crecimiento en conocimiento. Las universidades e instituciones similares tiene que hacer los ajustes necesarios para dominar las nuevas formas de un aprendizaje transformacional hecho posible por la revolución de las TIC, avanzando más allá de la tradicional tarea de transmitir información al más exigente rol de desarrollar las competencias para acceder, discriminar, analizar y utilizar el conocimiento. Más que nunca, estas instituciones tienen el deber de enseñar a los estudiantes como pensar creativamente para dominar grandes flujos de información en la búsqueda de soluciones.

4. Adaptar localmente pero manejar los recursos globalmente de un modo que confiera capacidad para enfrentar de manera flexible los retos locales mientras se utiliza el conocimiento global, la experiencia y los recursos compartidos, incluyendo los miembros de la facultad, el currículo, materiales didácticos y estudiantes enlazados internacionalmente mediante programas de intercambio.

5. Refuerzo de recursos educativos, ya que la facultad, sílabos, materiales didácticos e infraestructura son instrumentos necesarios para alcanzar competencias. Muchos países tiene graves déficits que requieren movilizar recursos, tanto financieros como didácticos, incluyendo el acceso abierto a jornales y materiales de enseñanza. El desarrollo de la facultad necesita especial atención mediante la creciente inversión en la educación de los educadores, rutas de carrera estables y gratificantes y una evaluación constructiva enlazada a incentivos por buen rendimiento.

6. Promover un nuevo profesionalismo que utilice competencias como el criterio objetivo para la clasificación de los profesionales de la salud, transformando los actuales silos convencionales. Un conjunto de actitudes, valores y conductas comunes se deberá desarrollar como la fundación para la preparación de una nueva generación de profesionales que complementen su aprendizaje de especialidades de experto son sus roles como agentes responsables del cambio, competentes administradores de recursos y promotores de políticas basadas en evidencia. Las reformas institucionales deberán estar en línea con los esfuerzos nacionales mediante la planificación conjunta, especialmente en los sectores de educación y salud, comprometer a todos los actores en el proceso de reforma, extender los ambientes de aprendizaje académico dentro de las comunidades, desarrollar redes globales colaborativas para el reforzamiento mutuo y el avance en la promoción de la cultura del cuestionamiento crítico y el razonamiento público.

7. El establecimiento de mecanismos de planificación conjunta en cada país para comprometer a los actores clave, especialmente ministros de educación y de salud, asociaciones profesionales y la comunidad académica, para superar la fragmentación mediante la evaluación de las condiciones nacionales, estableciendo prioridades, políticas de formación, seguimiento del cambio y armonizando el suministro y la demanda de profesionales de la salud que satisfagan las necesidades de salud de la población. En este proceso de planificación, especial atención se debe prestar al sexo y la geografía. A medida que la proporción de mujeres en la fuerza de trabajo en salud se incrementa, se necesita presentar iguales oportunidades-ej., mediante acuerdos de trabajo más flexibles, rutas de carrera que acomoden descansos temporales, el apoyo a otros roles sociales de la mujer tales como la crianza de los niños y una posición activa contra cualquier forma de discriminación o subordinación sexual. Con respecto a la distribución geográfica, se deberá poner énfasis en el reclutamiento de estudiantes de áreas marginales, ofreciendo incentivos financieros y de carrera a los proveedores que atienden estas áreas y desplegando el poder de TI para facilitar el aislamiento profesional.

8. La expansión de centros académicos a sistemas académicos, extendiendo el tradicional continuo de descubrimiento-cuidado-educación en las escuelas y hospitales hacia ubicaciones de atención primaria y comunidades, reforzada mediante la colaboración externa como parte de sistemas de educación profesional de mejor respuesta y dinámica.

9. Enlazar mediante redes, alianzas y consorcios entre instituciones educativas a nivel mundial y a través de todos los actores, tales como los gobiernos, las organizaciones de la sociedad civil, los negocios y los medios. En vista de la escasez de miembros de la facultad y otras limitaciones de recursos, cada país en desarrollo tiene pocas probabilidades de entrenar por si sola el completo complemento de profesionales de la salud que se requiere. Por lo tanto, se necesita establecer un consorcio regional y global como parte del diseño institucional en el siglo XXI, aprovechando las tecnologías de información y comunicación. La meta es superar las limitaciones de las instituciones individuales y expandir los recursos en el conocimiento, información y solidaridad para las misiones compartidas. Estas relaciones deberían estar basadas en los principios de compartir equitativamente los recursos sin explotación y sin paternalismos para generar el mutuo beneficio y responsabilidad.

10.Alimentar una cultura de cuestionamiento crítico como función central de las universidades y otras instituciones de enseñanza superior, lo cual es crucial para movilizar el conocimiento científico, la deliberación ética y el razonamiento y debate público para generar una progresista transformación social. 
a partir de la data recolectada bajo la dirección de los copresidentes y un equipo de investigación de apoyo.

\section{Fuentes de Financiamiento}

El trabajo de la Comisión fue apoyado mediante el financiamiento de Bill \& Melinda Gates Foundation, Rockefeller Foundation, y China Medical Board.

\section{Conflictos de Interés}

Los autores declaran no tener conflictos de interés.

Este reporte ha sido publicado en su versión en ingles en la revista The Lancet (Referencia original: Frenk J, Chen L, Bhutta ZA, et al. Health professionals for a new century: transforming education to strengthen health systems in an interdependent world The Lancet 2010; 376:1923-58). Esta traducción ha sido autorizada y reconoce los derechos de autor de la revista The Lancet.

Correspondencia: Patricia J. García

Dirección: Facultad de Salud Pública y Administración

Universidad Peruana Cayetano Heredia

Av. Honorio Delgado 430. San Martín de Porres. Lima 31, Perú.

Teléfono: (511) 319-0028

Correo electrónico: patricia.garcia@upch.pe

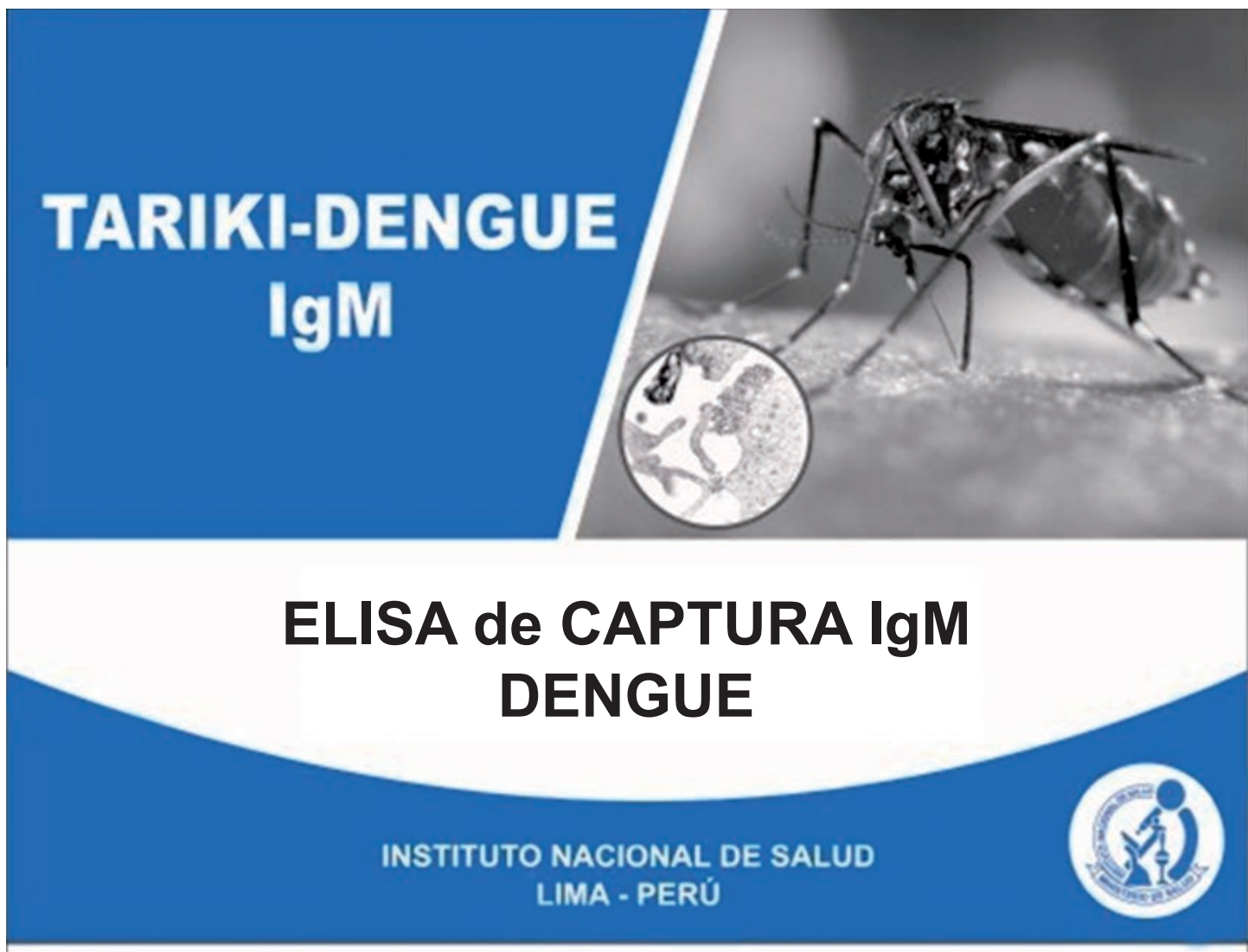

\section{Pronto disponible en el Perú....}

Kit para la determinación de anticuerpos IgM anti Dengue, desarrollado y producido por el Instituto Nacional de Salud. INS/MINSA 